\title{
Results From the 2014 November 15th Multi-chord Stellar Occultation by the Tno (229762) 2007 Uk 126
}

\section{Citation}

Benedetti-Rossi, G., B. Sicardy, M. W. Buie, J. L. Ortiz, R. Vieira-Martins, J. M. Keller, F. BragaRibas, et al. 2016. "RESULTS FROM THE 2014 NOVEMBER 15TH MULTI-CHORD STELLAR OCCULTATION BY THE TNO (229762) 2007 UK126." The Astronomical Journal 152 (6): 156. https://doi.org/10.3847/0004-6256/152/6/156.

\section{Permanent link}

http://nrs.harvard.edu/urn-3:HUL.InstRepos:41412162

\section{Terms of Use}

This article was downloaded from Harvard University's DASH repository, and is made available under the terms and conditions applicable to Other Posted Material, as set forth at http:// nrs.harvard.edu/urn-3:HUL.InstRepos:dash.current.terms-of-use\#LAA

\section{Share Your Story}

The Harvard community has made this article openly available. Please share how this access benefits you. Submit a story.

Accessibility 


\title{
RESULTS FROM THE 2014 NOVEMBER 15TH MULTI-CHORD STELLAR OCCULTATION BY THE TNO (229762) $2007 \mathrm{UK}_{126}$
}

\author{
G. Benedetti-Rossi ${ }^{1}$, B. Sicardy ${ }^{2}$, M. W. Buie ${ }^{3}$, J. L. Ortiz ${ }^{4}$, R. Vieira-Martins ${ }^{1,5}$, J. M. Kellek ${ }^{6}$, F. Braga-Ribas ${ }^{7}$, \\ J. I. B. Camargo ${ }^{1,8}$, M. Assafin ${ }^{5}$, N. Morales ${ }^{4}$, R. Duffard ${ }^{4}$, A. Dias-Oliveira ${ }^{1}$, P. Santos-Sanz ${ }^{4}$, J. Desmars ${ }^{9}$, \\ A. R. Gomes-Júnior ${ }^{5}$, R. LeIVA ${ }^{2,10}$, J. BARDeCKeR ${ }^{11,12}$, J. K. BeAN JR. ${ }^{12}$, A. M. Olsen ${ }^{11}$, \\ D. W. Ruby ${ }^{12,13}$, R. Sumner ${ }^{12}$, A. Thirouin ${ }^{14}$, M. A. Gómez-Muñoz ${ }^{15}$, L. Gutierrez ${ }^{15}$, L. Wasserman $^{14}$, \\ D. Charbonneau ${ }^{16}$, J. Irwin ${ }^{16}$, S. Levine ${ }^{14}$, and B. SkifF ${ }^{14}$ \\ ${ }^{1}$ Observatório Nacional_ON/MCT\&I, Brazil; gustavorossi@on.br, gugabrossi@gmail.com \\ ${ }^{2}$ LESIA, Observatoire de Paris, CNRS UMR 8109, Université Pierre et Marie Curie, Université Paris-Diderot, Meudon, France \\ ${ }^{3}$ Southwest Research Institute, Boulder, CO, USA \\ ${ }^{4}$ Instituto de Astrofísica de Andalucía, IAA-CSIC, Apt 3004, E-18080 Granada, Spain \\ ${ }^{5}$ Observatório do Valongo-OV/UFRJ, Brazil \\ ${ }^{6}$ California Polytechnic State University, San Luis Obispo, CA, USA \\ ${ }^{7}$ Universidade Tecnológica Federal do Paraná-UTFPR-DAFIS, Brazil \\ ${ }^{8}$ Laboratório Interinstitucional de e-Astronomia-LIneA, Rio de Janeiro, Brazil \\ ${ }^{9}$ IMCCE, Observatoire de Paris, PSL Research University, CNRS, Sorbonne Universités, UPMC, \\ Univ. Lille 1, 77 Av. Denfert-Rochereau, F-75014 Paris, France \\ ${ }^{10}$ Instituto de Astrofsica, Facultad de Fsica, Pontificia Universidad Catlica de Chile, Santiago 7820436, Chile \\ ${ }^{11}$ IOTA, International Occultation Timing Association, USA \\ ${ }^{12}$ RECON, Research and Education Collaborative Occultation Network, USA \\ ${ }_{14}^{13}$ University of Nevada, Reno, NV, USA \\ ${ }^{14}$ Lowell Observatory, Flagstaff, AZ, USA \\ ${ }^{15}$ Instituto de Astronomía-Universidad Nacional Autónoma de México-UNAM, Mexico \\ ${ }^{16}$ Harvard-Smithsonian Center for Astrophysics, MA, USA \\ Received 2016 April 12; revised 2016 July 28; accepted 2016 July 28; published 2016 November 11
}

\begin{abstract}
We present results derived from the first multi-chord stellar occultation by the trans-Neptunian object (229762) 2007 UK $_{126}$, observed on 2014 November 15. The event was observed by the Research and Education Collaborative Occultation Network project and International Occultation Timing Association collaborators throughout the United States. Use of two different data analysis methods obtain a satisfactory fit to seven chords, yielding an elliptical fit to the chords with an equatorial radius of $R=338_{-10}^{+15} \mathrm{~km}$ and equivalent radius of $R_{\mathrm{eq}}=319_{-7}^{+14} \mathrm{~km}$. A circular fit also gives a radius of $R=324_{-23}^{+30} \mathrm{~km}$. Assuming that the object is a Maclaurin spheroid with indeterminate aspect angle, and using two published absolute magnitudes for the body, we derive possible ranges for geometric albedo between $p_{V}=0.159_{-0.013}^{+0.007}$ and $p_{R}=0.189_{-0.015}^{+0.009}$, and for the body oblateness between $\epsilon=0.105_{-0.040}^{+0.050}$ and $\epsilon=0.118_{-0.048}^{+0.055}$. For a nominal rotational period of $11.05 \mathrm{hr}$, an upper limit for density of $\rho=1740 \mathrm{~kg} \mathrm{~m}^{-3}$ is estimated for the body.
\end{abstract}

Key words: Kuiper belt objects: individual (229762, 2007 UK126) - occultations -

planets and satellites: fundamental parameters

\section{INTRODUCTION}

Trans-Neptunian objects (TNOs) are remnants of a collisionally and dynamically evolved planetesimal disk in the outer solar system. Their physical characteristics can provide and reveal important clues about the primordial protoplanetary nebula, planet formation, and other evolutionary processes (Lykawka \& Mukai 2008). Moreover, the inferred chemical, thermal, and collisional processes that they underwent tell us something about the evolution of the outer solar system. However, their large distances make the study of those bodies difficult, and our knowledge about their sizes, shapes, albedo, densities, and atmospheres remains fragmentary (Stansberry et al. 2008; Parker et al. 2015).

In the past 25 years, more than 1900 TNOs and Centaurs have been discovered (Minor Planet Center 2016a, 2016b). The stellar occultation technique is a very accurate tool to study those bodies, as it provides sizes and shapes at the kilometer level, can detect atmospheres at the nanobar level (Sicardy et al. 2011; Ortiz et al. 2012), and is even sensitive to features such as jets and rings (Braga-Ribas et al. 2014; Ortiz et al.
2015). Since 2009, after the first successful observation of a stellar occultation by a TNO (other than Pluto or Charon) called $2002 \mathrm{TX}_{300}$ (Elliot et al. 2010), several objects have been observed by stellar occultations. Examples are Varuna (Sicardy et al. 2010), Eris (Sicardy et al. 2011), 2003 AZ $_{84}$ (Braga-Ribas et al. 2011; Braga-Ribas et al. 2012), Quaoar (Person et al. 2011; Sallum et al. 2011; Braga-Ribas et al. 2013), Makemake (Ortiz et al. 2012), and $2002 \mathrm{KX}_{14}$ (Alvarez-Candal et al. 2014), and Centaur objects like Chariklo (Braga-Ribas et al. 2014) and Chiron (Ortiz et al. 2015; Ruprecht et al. 2015). The observation of a multi-chord stellar occultation on 2014 November 15 increases that list to include the TNO (229762) $2007 \mathrm{UK}_{126}$, the main topic of this paper.

This TNO was discovered by Schwamb et al. (2008) in 2007 October with an estimated radius and albedo of $299.5 \pm 38.9 \mathrm{~km}$ and $0.167_{-0.038}^{+0.058}$ (Santos-Sanz et al. 2012), respectively. With a semimajor axis of $73.81 \mathrm{AU}$, aphelion distance of $109.7 \mathrm{AU}$, orbital period of $634.13 \mathrm{yr}$, orbital eccentricity of 0.492 , and an inclination of $23^{\circ} .34$ (JPL SmallBody Database Browser 2016), it is usually classified as a 
Scattered Disk Object (SDO, according to Gladman et al. 2008) or a member of the class of "Detached Objects" (see e.g., Lykawka \& Mukai 2008). Moreover, Grundy et al. (2011) reported the discovery of a companion with a magnitude difference of 3.79 mag in the F606W band of the Hubble Space Telescope. Its orbit is still unknown, but it is expected to be non-circular (Thirouin et al. 2014).

In this paper, we present results derived from the 2014 November 15 stellar occultation by this body. Section 2 briefly describes our prediction scheme and presents the observations. Data analysis is described in Section 3. The size and shape of the TNO as well as their physical implications are discussed in Section 4, before concluding remarks in Section 5.

\section{PREDICTIONS AND OBSERVATIONS}

The 2014 November 15 occultation was identified in a systematic search for TNO occultation candidate stars, made at the $2.2 \mathrm{~m}$ telescope of ESO, using the Wide Field Imager. This search yielded local astrometric catalogs for 5 Centaurs and 34 TNOs (plus Pluto and its moons) up to 2015, and for stars with magnitudes as faint as $R \sim 19$. Further details can be found in Assafin et al. (2010), Assafin et al. (2012), Camargo et al. (2014), and Desmars et al. (2015).

After identifying the target star, astrometric updates of the star UCAC4 448-006503 (UCAC2 31623811, $R=15.7$ ) close in time to the predicted occultation were performed with the $60 \mathrm{~cm}$ telescope at Pico dos Dias Observatory (OPD/LNAIAU code 874) and with the $77 \mathrm{~cm}$ telescope at La Hita Observatory (IAU code I95). From OPD, 20 images with $45 \mathrm{~s}$ exposure time were acquired using Johnson's I filter (centered at $800 \mathrm{~nm}$ ) and an IkonL 9867 CCD camera on 2014 October 19. From La Hita, 99 unfiltered images of $400 \mathrm{~s}$ exposure time were obtained on 2014 October 29-31, with the $4 \mathrm{k} \times 4 \mathrm{k}$ camera, which provided a very wide field of view of $47 \times 47$ arcmin. In both cases, the images were obtained at times when the objects were near the meridian, to minimize possible Differential Chromatic Refraction (DCR) problems. Unfortunately, since the apparent visual magnitude of the TNO is approximately 20.1 , the signal-to-noise ratio of $2007 \mathrm{UK}_{126}$ in the individual exposures was poor (around 4-8, depending mainly on the seeing) and did not allow us to obtain more accurate astrometry of the TNO.

To derive accurate astrometry of $2007 \mathrm{UK}_{126}, 36$ images of the TNO were obtained with the Calar Alto (IAU code 493) $1.2 \mathrm{~m}$ telescope using the $4 \mathrm{k} \times 4 \mathrm{k}$ DLR CCD camera on 2014 October 28 and 29. The camera provides a field of view of $22 \times 22$ arcmin. Exposure times were $400 \mathrm{~s}$, which allowed us to obtain a signal-to-noise ratio on the target larger than 40 , with no filter. The images were also obtained when the object was near culmination to minimize any possible problem due to DCR. The astrometry provided the offsets in right ascension and declination with respect to the nominal positions based on the JPL Horizons ephemeris. From the dispersion of the offset measurements (i.e., the quadratic sum of the $1-\sigma$ uncertainty in the ephemeris update (20 mas) and the $1-\sigma$ error in the position of the star (4 mas)) the final uncertainty in the prediction was estimated at about 20.4 mas, comparable to the expected shadow path width. The final prediction indicated that the shadow was favorable for observers in several states in the USA (Figure 1). A compilation of our measurements provides the following ICRF/J2000 star position at the date of the occultation:

$$
\begin{gathered}
\alpha=+04^{\mathrm{h}} 29^{\mathrm{m}} 30^{\mathrm{s}} .6100 \pm 0 .^{\prime \prime} 022 \\
\delta=-00^{\circ} 28^{\prime} 20 !^{\prime \prime} 908 \pm 0.023 .
\end{gathered}
$$

The Research and Education Collaborative Occultation Network (RECON) project (Buie \& Keller 2016) pilot sites and other potential sites participated in the campaign for a total of 20 different stations (Tables 1 and 2). Bad weather conditions spoiled observations in 11 sites. Meanwhile, six sites from RECON, one International Occultation Timing Association (IOTA) site located in Urbana, Illinois, and two telescopes at San Pedro Martir acquired data, for a total of seven positive detections of the event and two negative chords at San Pedro Martir, which were located more than $500 \mathrm{~km}$ south of the shadow path (Figure 1). The times of the star disappearances (ingress) and re-appearances (egress) for the seven detections are listed on Table 3.

All acquired data, with exception of the data from the two telescopes in San Pedro Martir, were in video format. A video time inserter (VTI) was used to place a time-stamp on each individual video frame. All RECON sites use IOTA-VTI that The VTI interacts with a GPS receiver to obtain the time, ideally with an absolute accuracy of a few milliseconds, and then superimpose the current time on each video field as it passes from the camera to the computer (see Buie \& Keller 2016). Unfortunately, no information is saved on any image header because of the video format.

\section{DATA ANALYSIS}

Considerable detail is provided on video data that is applicable to RECON data in Buie \& Keller (2016). In particular, the discussion on frame and field interleaving of the data is especially relevant for these data. All of the RECON data were collected with a SENSEUP value of $128 \times$, meaning that the integration time is equal to 128 times the field rate of the NTSC video signal, which resultted in integrations of approximately $2 \mathrm{~s}$. The Urbana data were collected with a Watec camera integrating 128 video frames, or approximately $4 \mathrm{~s}$. If there are no dropped frames or other problems, the RECON video will have 64 copies of each integration in the video data stream. The Urbana video will have 128 copies.

Two independent analyses were performed on the video data to extract light curves and timing information. The two approaches are different enough to be useful as a cross-check of results to provide information on the uncertainties in the final projected shape. The primary analysis is from Benedetti-Rossi (GBR) and the secondary analysis is from Buie (MWB).

\subsection{Occultation Light Curves}

\subsubsection{GBR Extraction}

Using AUDELA (a free and open source astronomy software for digital observations: CCD cameras, Webcams, etc.) we extracted individual frames to FITS format from the video at a rate of 29.97 frames per second. A careful check was done in all sets of images to verify whether or not the extracted time corresponds to the time printed at each frame.

The frames were then grouped to match the corresponding SENSEUP value. The first and last frames of each 64 frame (or 128) sequence were identified by counting frames from a calibrated starting point and checked with a change in field brightness. They were then excluded and the other 62 (or 126) 


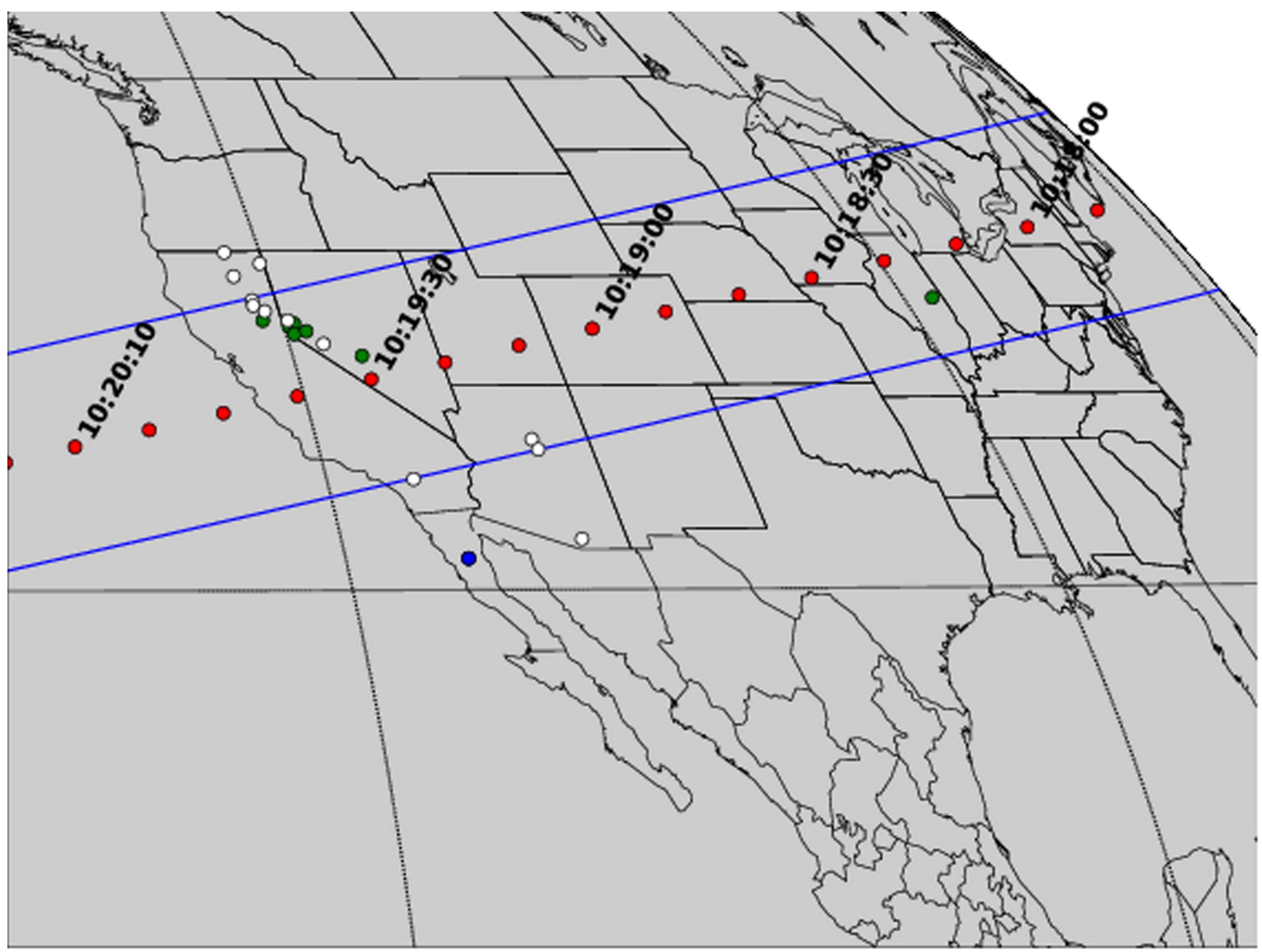

Figure 1. Post-occultation reconstruction of $2007 \mathrm{UK}_{126}$ 's shadow path on Earth for the 2014 November 15 event. The shadow moves from right to left; blue lines are the expected size limit of the TNO (kilometers), as derived from Figures 3 and 4; the red dots represents the center of the body for a given time, each separated by $10 \mathrm{~s}$. The green dots are the sites where the occultation was detected (Table 1). The blue dots are the two telescopes at San Pedro Martir, that acquired data but did not detect the event, and the white dots are the sites that were clouded out or suffered technical failures (Table 2).

frames were averaged to obtain each image that corresponds to an individual exposure time. By not considering the first and last frame from a sequence, we avoid the need to de-interlace and re-interlace the frames, and we do not take into account any field from the previous or next sequence. Note that this process also preserves the mid-time of each image, which was extracted from the mid-frame of each sequence. This method generates times with a systematic shift with respect to the absolute times (one integration cycle) but does not affect the final shape when all chords are processed the same way. This whole process of converting video to separate stack image files requires special attention because of possible dropped frames, duplicated fields, or any incompatibility with different software packages, drivers, or plug-ins.

Differential aperture photometry was extracted from the data using the PRAIA package (Assafin et al. 2011) to obtain the light curves. Two field stars with 8 pixel and a third one with 10 pixel photometric aperture were used to calibrate the occulted star flux. Sky background flux was obtained from an annulus of internal radius of 16 and external radius of 20 pixels around the first two calibration stars and 20-24 pixels around the third one. For the occulted star, a photometric aperture of 7 pixels was used while for the sky background flux an annulus of 10 pixels inner radius and 16 pixels for the outer radius was used.

The occulted star flux was then normalized to the unocculted stellar flux by applying a third-degree polynomial fit to the flux just before and after the event. The resulting light curves are shown in Figure 2.

\subsubsection{MWB Extraction}

Accurate determination of the time of each integration is discussed in detail in Buie \& Keller (2016) and requires locating the exact place in the video stream where a new integration is first seen.

In this analysis, custom software was created in IDL for each site's data. The first step requires converting the AVI-format video data to individual image files. The freely available tool ffmpeg, ${ }^{17}$ was used to extract a sequence of images to individual PNG format files. In all cases only 90-120 s of data were extracted, centered if possible on the occultation chord.

The basic flow of data processing contains some or all of the following steps, customized for each data set. (1) Create a mean sky image. If possible to build, this image contains the general background gradient, typically from amplifier glow in one corner as well as hot pixels. Sites where the tracking was perfect could not be corrected since separate dark or sky images were not collected. Mean sky images require a rather complicated stacking of frames. The first step is to perform a robust stack of one image per integration. There are as many of these stacks as there are frames in an integration and these stacks are also robustly averaged into the final mean sky image.

\footnotetext{
${ }^{17}$ https://www.ffmpeg.org/
} 
Table 1

Circumstances of Observation for Stations that Acquired Data

\begin{tabular}{|c|c|c|c|c|c|}
\hline Site & $\begin{array}{l}\text { Longitude (W) } \\
\text { Latitude (N) } \\
\text { Altitude (m) }\end{array}$ & $\begin{array}{l}\text { Telescope }^{\mathrm{a}} \\
\text { Camera }\end{array}$ & $\begin{array}{l}\text { Exposure } \\
\text { Time } \\
\text { (s) }\end{array}$ & $\begin{array}{l}\text { Light curve } \\
\text { rms } \\
\text { GBR/MWB }\end{array}$ & $\begin{array}{l}\text { Observer } \\
\text { Note }\end{array}$ \\
\hline Reno & $\begin{array}{l}119^{\circ} 45^{\prime} 53 !^{\prime \prime} 0 \\
39^{\circ} 23^{\prime} 28^{\prime \prime} 5 \\
1470\end{array}$ & $\begin{array}{l}\text { Standard RECON } \\
\text { hardware setup }^{\text {b }}\end{array}$ & 2 & $0.235 / 0.140$ & $\begin{array}{l}\text { Dan Ruby, Brian Crosby, } \\
\text { Seth Nuti } \\
\text { (RECON) }\end{array}$ \\
\hline Jack C. Davis Observatory & $\begin{array}{c}119^{\circ} 47^{\prime} 46^{\prime \prime} 8 \\
39^{\circ} 11^{\prime} 08^{\prime \prime} 2\end{array}$ & $\begin{array}{l}\text { Meade LX-200 } \\
30 \mathrm{~cm} \text { telescope }\end{array}$ & 2 & $0.212 / 0.194$ & Jim Bean, Ethan Lopes \\
\hline "Carson City (B)" & 1548.1 & & & & (RECON) \\
\hline Carson City (S) & $\begin{array}{c}119^{\circ} 33^{\prime} 31^{\prime \prime} 4 \\
39^{\circ} 16^{\prime} 26^{\prime \prime} 5 \\
1332.6\end{array}$ & $\begin{array}{l}\text { Standard RECON } \\
\text { hardware setup }\end{array}$ & 2 & $0.191 / 0.123$ & $\begin{array}{l}\text { Red Sumner } \\
\text { (RECON) }\end{array}$ \\
\hline Gardnerville & $\begin{array}{c}119^{\circ} 40^{\prime} 20^{\prime \prime} 3 \\
38^{\circ} 53^{\prime} 23^{\prime \prime} 5 \\
1534.9\end{array}$ & $\begin{array}{l}\text { Meade LX-200 } \\
30 \mathrm{~cm} \text { telescope } \\
\text { MallinCAM B\&W } 428\end{array}$ & 2 & $0.197 / 0.123$ & $\begin{array}{l}\text { Jerry Bardecker } \\
(\text { RECON) }\end{array}$ \\
\hline Yerington & $\begin{array}{c}119^{\circ} 09^{\prime} 39^{\prime \prime} 0 \\
38^{\circ} 59^{\prime} 28^{\prime \prime} 3 \\
1342.7\end{array}$ & $\begin{array}{l}\text { Standard RECON } \\
\text { hardware setup }\end{array}$ & 2 & $0.205 / 0.131$ & $\begin{array}{l}\text { Todd Hunt, Scott Darrington, } \\
\text { Joanna Kuzia, Les Kuzia, } \\
\text { Matt Christiansen } \\
\text { (RECON) }\end{array}$ \\
\hline Tonopah & $\begin{array}{c}117^{\circ} 14^{\prime} \quad 06^{\prime \prime} 7 \\
38^{\circ} 05^{\prime} 22^{\prime \prime} 1 \\
1838.7\end{array}$ & $\begin{array}{l}\text { Standard RECON } \\
\text { hardware setup }\end{array}$ & 2 & $0.214 / 0.128$ & $\begin{array}{l}\text { Teralyn Blackburn, } \\
\text { Clair Blackburn } \\
\text { (RECON) }\end{array}$ \\
\hline Urbana & $\begin{array}{c}088^{\circ} 11^{\prime} 46^{\prime \prime} 4 \\
40^{\circ} 05^{\prime} 12^{\prime \prime} 5 \\
227\end{array}$ & $\begin{array}{l}50 \mathrm{~cm} \text { Newtonian } \\
\text { Watec-120N+ }\end{array}$ & 4 & $0.180 / 0.168$ & Aart Olsen \\
\hline San Pedro Martir & $\begin{array}{l}115^{\circ} 27^{\prime} 58^{\prime \prime} 0 \\
31^{\circ} 02^{\prime} 42^{\prime \prime} 0 \\
2790\end{array}$ & $\begin{array}{l}\text { OAN/SPM Harold } \\
\text { L. Johnson } 1.5 \mathrm{~m} \text { telescope } \\
\text { FLI ProLine } \\
\text { PL3041 (PL0212309) }\end{array}$ & 2 & $\cdots$ & Leonel Gutierrez et al. \\
\hline San Pedro Martir & $\begin{array}{l}115^{\circ} 28^{\prime} 00 !^{\prime \prime} 0 \\
31^{\circ} 02^{\prime} 433^{\prime \prime} 0 \\
2790\end{array}$ & $\begin{array}{l}0.84 \mathrm{~m} \text { telescope } \\
\text { SPECTRAL E2V-4240 } \\
\text { Mexman }\end{array}$ & 5 & $\ldots$ & Marco Gómez et al. \\
\hline
\end{tabular}

Notes.

${ }^{\mathrm{a}}$ For more details on the RECON equipment, see Buie \& Keller (2016).

b Standard RECON hardware setup consist in a $28 \mathrm{~cm}$ Celestron CPC1100 telescope and a MallinCAM B\&W 428.

If the entire cube is stacked at once, the frame replication for the integrations will subvert the robust averaging algorithm. (2) Subtract a mean sky image from each frame. (3) Re-interlace the images, if needed. The need for re-interlacing is easily seen in a raw light curve of a bright star. Each integration has a unique signal level and will look like steps from one integration to the next. If the signal steps cleanly between integrations, the interlacing is correct. If there is a single frame point between the two levels, re-interlacing is required. (4) Extract source and comparison star fluxes from each frame. (5) Down-sample by averaging to a single measurement for each integration. (6) Apply timing formula from Buie \& Keller (2016) to get absolute timing of each data point. The deviations from this set of steps is now described for each site in turn.

The photometric extraction required some special handling in all cases. Four nearby field stars were measured to obtain both position and flux. When the occultation star was visible, its position was also measured. The offset for the occultation star relative to the brightest field star was determined for each data set. This offset was used as the exact position for extracting the occultation star flux on each image. This process avoids aperture wander off of the occultation star location during the occultation. Each site reduction process is presented as follows.

Ruby/Reno-A mean sky frame was generated and subtracted. The data were re-interlaced and had no dropped frames. The photometry was generated from a 5 pixel object aperture and a sky annulus from 8-40 pixels. The raw photometry shows clear signs of degrading sky conditions from 10:18-10:20 UT. Getting the timing required a more careful examination of the images and determination of the brightness of the transitional frames for the model timing extractions.

Sumner/Carson City (S) — A sky frame was generated and subtracted. The images were re-interlaced and had the same dropped frame problem found with the other Carson City site. The final results have full-quality data after cleanup. The photometry was generated with a 5 pixel object aperture and the sky was determined from a robust mean of the entire image which was flat due to the subtracted sky mean.

Jack C. Davis Observatory/Carson City (B) - The mean sky image could not be generated to high-quality track and no calibration images. The data required re-interlacing and also 
Table 2

Circumstances of Observation for Stations with No Data Acquired

\begin{tabular}{|c|c|c|c|c|}
\hline Site & $\begin{array}{l}\text { Longitude }(\mathrm{W}) \\
\text { Latitude }(\mathrm{N}) \\
\text { Altitude (m) }\end{array}$ & $\begin{array}{l}\text { Telescope }^{\mathrm{a}} \\
\text { Camera }\end{array}$ & Result & $\begin{array}{l}\text { Observer } \\
\text { Note }\end{array}$ \\
\hline $\begin{array}{l}\text { Lowell Observatory } \\
\text { Anderson Mesa }\end{array}$ & $\begin{array}{c}111^{\circ} 32^{\prime} 09^{\prime \prime} 0 \\
35^{\circ} 05^{\prime} 49^{\prime \prime} 0 \\
2163\end{array}$ & $\begin{array}{l}1.1 \mathrm{~m} \text { Hall } \\
\text { nasa42 }\end{array}$ & Clouds & Brian Skiff \\
\hline Cedarville & $\begin{array}{l}120^{\circ} 09^{\prime} 9^{\prime \prime} 4 \\
41^{\circ} 31^{\prime} 50^{\prime \prime} 0 \\
1381.4\end{array}$ & $\begin{array}{l}\text { Standard RECON } \\
\text { hardware setup }\end{array}$ & Clouds & $\begin{array}{l}\text { Brian Cain, Terry Miller, } \\
\text { David Schulz } \\
\text { (RECON) }\end{array}$ \\
\hline $\begin{array}{l}\text { CPSLO } \\
\text { Idyllwild/Astrocamp }\end{array}$ & $\begin{array}{c}116^{\circ} 42^{\prime} 48^{\prime \prime} 9 \\
33^{\circ} 44^{\prime} 33^{\prime \prime} 4 \\
1714.2\end{array}$ & $\begin{array}{l}\text { Meade LX-200 } \\
30 \mathrm{~cm} \text { telescope } \\
\text { MallinCAM B\&W } 428\end{array}$ & $\begin{array}{l}\text { Clouds and } \\
\text { Focus } \\
\text { problems }\end{array}$ & $\begin{array}{l}\text { John Keller, Melanie Phillips, } \\
\text { Eric Hsieh, Ian Mahaffey, } \\
\text { Tedd Zel, Jeff Schloetter, } \\
\text { Andrew Yoder, Jiawei Simon Qin } \\
\text { Jacob Wagner, Adam Eisenbarth } \\
\text { (RECON) }\end{array}$ \\
\hline $\begin{array}{l}\text { Lowell Observatory } \\
\text { Discovery Channel } \\
\text { Telescope }\end{array}$ & $\begin{array}{c}111^{\circ} 25^{\prime} 20^{\prime \prime} 0 \\
34^{\circ} 44^{\prime} 40^{\prime \prime} 0 \\
2360\end{array}$ & $\begin{array}{l}4.3 \mathrm{~m} \\
\text { Large Monolith Imager }\end{array}$ & Clouds & Stephen Levine \\
\hline Fall River/Burney & $\begin{array}{c}121^{\circ} 23^{\prime} 56^{\prime \prime} \\
41^{\circ} 02^{\prime} 45^{\prime \prime} \\
1012\end{array}$ & $\begin{array}{l}\text { Standard RECON } \\
\text { hardware setup }\end{array}$ & Clouds & $\begin{array}{l}\text { Andrew Mayncsik } \\
(\mathrm{RECON})\end{array}$ \\
\hline $\begin{array}{l}\text { Fred Lawrence } \\
\text { Whipple Observatory }\end{array}$ & $\begin{array}{c}110^{\circ} 52^{\prime} 42^{\prime \prime} 0 \\
31^{\circ} 40^{\prime} 52^{\prime \prime} 0 \\
2606\end{array}$ & MEarth & Clouds & $\begin{array}{l}\text { J. Irwin, } \\
\text { D. Charbonneau }\end{array}$ \\
\hline Greenville & $\begin{array}{c}120^{\circ} 57^{\prime} 04^{\prime \prime} \\
40^{\circ} 08^{\prime} 23^{\prime \prime} \\
1098\end{array}$ & $\begin{array}{l}\text { Standard RECON } \\
\text { hardware setup }\end{array}$ & Clouds & $\begin{array}{l}\text { Bill Gimple, Barclay Anderson } \\
(\text { RECON) }\end{array}$ \\
\hline Hawthorne & $\begin{array}{c}118^{\circ} 37^{\prime} 49^{\prime \prime} \\
38^{\circ} 31^{\prime} 35^{\prime \prime} \\
1321\end{array}$ & $\begin{array}{l}\text { Standard RECON } \\
\text { hardware setup }\end{array}$ & $\begin{array}{l}\text { Instrument } \\
\text { problems }\end{array}$ & $\begin{array}{l}\text { Kathy Trujillo } \\
\text { (RECON) }\end{array}$ \\
\hline Portola & $\begin{array}{c}120^{\circ} 34^{\prime} 03^{\prime \prime} 5 \\
39^{\circ} 43^{\prime} 56^{\prime \prime} 1 \\
1351\end{array}$ & $\begin{array}{l}\text { Standard RECON } \\
\text { hardware setup }\end{array}$ & Frost/Clouds & $\begin{array}{l}\text { Shelley Callahan, } \\
\text { Mark Callahan } \\
\text { (RECON) }\end{array}$ \\
\hline $\begin{array}{l}\text { Quincy } \\
\text { Feather River College }\end{array}$ & $\begin{array}{c}120^{\circ} 58^{\prime} 10^{\prime \prime} 3 \\
39^{\circ} 56^{\prime} 52^{\prime \prime} 7 \\
1046\end{array}$ & $\begin{array}{l}\text { Standard RECON } \\
\text { hardware setup }\end{array}$ & Clouds & $\begin{array}{l}\text { Charley Arrowsmith, Lynn Coffman, } \\
\text { Levi Kinateder, Colton Kohler, } \\
\text { Mystery Brown } \\
\text { (RECON) }\end{array}$ \\
\hline Susanville & $\begin{array}{c}119^{\circ} 45^{\prime} 533^{\prime \prime} 0 \\
39^{\circ} 23^{\prime} 28 !^{\prime \prime} 4 \\
1470\end{array}$ & $\begin{array}{l}\text { Meade LX-200 } \\
30 \mathrm{~cm} \text { telescope } \\
\text { MallinCAM B\&W } 428\end{array}$ & Clouds & $\begin{array}{l}\text { Buck Bateson } \\
\text { (RECON) }\end{array}$ \\
\hline Tulelake & $\begin{array}{c}121^{\circ} 28^{\prime} 44^{\prime \prime} \\
41^{\circ} 57^{\prime} 19^{\prime \prime} \\
1232\end{array}$ & $\begin{array}{l}\text { Standard RECON } \\
\text { hardware setup }\end{array}$ & Clouds & $\begin{array}{l}\text { Jason Matkins, Jeannie Smith } \\
\text { (RECON) }\end{array}$ \\
\hline
\end{tabular}

Note.

${ }^{\mathrm{a}}$ For more details on the RECON equipment, see Buie \& Keller (2016).

suffered from dropped frames. Each frame was manually inspected to identify corrupted frames (one or two every $3 \mathrm{~s}$ ). During the manual inspection the IOTA-VTI timing information was used to establish the identity of each frame and it's association with the individual integrations. In some cases, all 64 frames were good, but many had one or two frames that were not used. When properly identified, the timing is not affected by the loss of a few frames out of the 64 copies that should have been collected. In no case was there a dropped frame on an integration boundary. The photometric used was a
5 pixel object aperture and a sky annulus of 8-25 pixels. The final result, though laborious, was not affected by the dropped frames.

Yerington-A mean sky frame was generated and subtracted. The data were re-interlaced and had no dropped frames. The photometry was generated from a 5 pixel object aperture and sky was determined from the mean of each frame.

Bardecker/Gardnerville-The mean sky image could not be generated due to the excellent tracking at this site and the lack of separate calibration image sequences. The video data 
Table 3

Disappearance (ingress) and Reappearance (egress) Times

\begin{tabular}{|c|c|c|c|c|c|}
\hline Site & $\begin{array}{c}\text { GBR } \\
\text { Ingress (UTC) } \\
\text { Egress (UTC) }\end{array}$ & $\begin{array}{l}\text { Error } \\
(\mathrm{s})\end{array}$ & $\begin{array}{c}\text { MWB } \\
\text { Ingress (UTC) } \\
\text { Egress (UTC) }\end{array}$ & $\begin{array}{l}\text { Error } \\
(\mathrm{s})\end{array}$ & $\begin{array}{c}\text { Difference }^{\mathrm{a}} \\
\text { (s) }\end{array}$ \\
\hline \multirow[t]{2}{*}{ Reno } & $10: 19: 35.02$ & 0.80 & $10: 19: 34.10$ & 0.70 & 0.92 \\
\hline & $10: 19: 46.68$ & 0.47 & $10: 19: 46.64$ & 0.65 & 0.04 \\
\hline \multirow[t]{2}{*}{ Carson City (S) } & $10: 19: 30.85$ & 0.66 & $10: 19: 30.60$ & 0.60 & 0.25 \\
\hline & $10: 19: 46.32$ & 0.41 & $10: 19: 46.30$ & 0.42 & 0.02 \\
\hline \multirow[t]{2}{*}{ Carson City (B) } & $10: 19: 32.47$ & 0.51 & $10: 19: 32.20$ & 0.50 & 0.27 \\
\hline & $10: 19: 47.19$ & 0.35 & $10: 19: 47.30$ & 0.41 & -0.11 \\
\hline \multirow[t]{2}{*}{ Yerington } & $10: 19: 29.42$ & 0.40 & $10: 19: 29.25$ & 0.41 & 0.17 \\
\hline & $10: 19: 45.97$ & 0.35 & $10: 19: 46.00$ & 0.35 & -0.03 \\
\hline \multirow[t]{2}{*}{ Gardnerville } & $10: 19: 29.40$ & 0.42 & 10:19:29.71 & 0.46 & -0.31 \\
\hline & $10: 19: 48.35$ & 0.29 & $10: 19: 48.07$ & 0.30 & 0.28 \\
\hline \multirow[t]{2}{*}{ Tonopah } & $10: 19: 17.49$ & 0.54 & $10: 19: 17.60$ & 0.55 & 0.11 \\
\hline & $10: 19: 42.39$ & 0.26 & $10: 19: 42.45$ & 0.30 & -0.06 \\
\hline \multirow[t]{2}{*}{ Urbana } & 10:18:03.76 & 0.90 & 10:18:03.61 & 0.90 & 0.15 \\
\hline & $10: 18: 27.62$ & 0.90 & $10: 18: 27.60$ & 0.90 & 0.02 \\
\hline
\end{tabular}

Note.

a Difference between times in the sense "GBR-MWB."

required re-interlacing but did not have any instances of dropped frames in the video data stream. A 5 pixel photometric aperture was used for the sources and a local sky value was determined for each with a sky annulus of 8-40 pixels. The frame integration boundary was visually determined by seeing the change in the background noise. A single transition was sufficient to establish the timing for the entire sequence. Note that this site used a telescope with an equatorial mount.

Tonopah-A mean sky frame was generated and subtracted. The data were re-interlaced and had no dropped frames. The photometry was generated from a 5 pixel object aperture and the sky was determined from the mean of each frame.

Olsen/Urbana-This data set uses a similar but not identical setup to the standard RECON system. The camera was a Watec-120N+ and has a similar sensitivity and operation to the RECON MallinCAM cameras but can integrate twice as long. The timing was provided by a Kiwi OSD VTI. A sky frame was generated and subtracted. The images were re-interelaced and there were no dropped frames. A 5 pixel photometric aperture was used with a sky annulus of 8-50 pixels to remove the small amount of flat sky residual background.

All the resulting light curves are shown in Figure 2.

\subsection{Occultation Timing}

As described by Braga-Ribas et al. (2013), the start and end times of the occultation were obtained for each light curve by fitting a sharp edge occultation model. This model is convolved by Fresnel diffraction, the CCD bandwidth, the stellar apparent angular diameter in kilometers, and the finite integration time (see Widemann et al. 2009 for more details).

The Fresnel scale $(F=\sqrt{\lambda D / 2})$ for the geocentric distance $D=42.6 \mathrm{AU}$ (or $6.37 \times 10^{9} \mathrm{~km}$ ) of $2007 \mathrm{UK}_{126}$ by the time of the event is approximately $1.4 \mathrm{~km}$ for a typical wavelength of $\lambda=0.65 \mu \mathrm{m}$. The star apparent angular diameter is estimated using the formulae of van Belle (1999). Its $B, V$, and $K$ apparent magnitudes are 16.2, 15.6, and 13.7, respectively, in the NOMAD catalog (Zacharias et al. 2004). This yields a diameter of about $0.3 \mathrm{~km}$ projected at the distance of the $2007 \mathrm{UK}_{126}$. The smallest integration time used in the positive observations was $2 \mathrm{~s}$, which translates to almost $48 \mathrm{~km}$ in the celestial plane. Therefore, the occultation light curves are largely dominated by the integration times, not by Fresnel diffraction or the star diameter.

The occultation fits consist of minimizing a classical $\chi^{2}$ function for each light curve, as described in Sicardy et al. (2011). The free parameter to adjust is the ingress (disappearance) or egress (reappearance) time, which provides the minimum value of $\chi^{2}$ denoted as $\chi_{\min }^{2}$. The best fits to the occultation light curves are shown in Figure 2, and the derived instants of ingress and egress are shown in Table 3.

Note in Figure 2 that the disappearance and reappearance of the star is very clear with exception of the Reno observation. The Reno data required special care due to the degrading sky conditions after 10:18 UT. At first glance, the point in the light curve near 10:19:32 seems to indicate the start of the occultation. However, careful examination of this integration still shows a faint remnant of the star along with some image artifacts and leads to an anomalously low signal. The integration at 10:19:34 clearly shows the star and is the frame where ingress begins as the star is completely gone by the next integration. In general, one might think the point at 10:19:32 is perhaps indicating some other interesting light-curve feature other than ingress. In this case, the degrading observing conditions argue that the measured flux from this integration is spurious and should be treated as an unocculted timestep, and that is what we adopt for the interpretation of those light curves.

\subsection{Limb Fitting}

Objects with diameter larger than about $1000 \mathrm{~km}$ are expected to be in hydrostatic equilibrium. As such, they reach either Maclaurin spheroid or Jacobi ellipsoid states (Chandrasekhar 1987). The critical diameter, defined as the minimum size necessary to reach hydrostatic equilibrium, can be estimated to $200-900 \mathrm{~km}$ for icy bodies or from $500-1200 \mathrm{~km}$ for rocky bodies (Tancredi \& Favre 2008). $2007 \mathrm{UK}_{126}$ is within those critical ranges and considering it is in the small angular momentum regime (i.e., the body presents a low rotation period with an estimated rotational period of $11.05 \mathrm{hr}$ and it presents a small-amplitude rotational light curve 

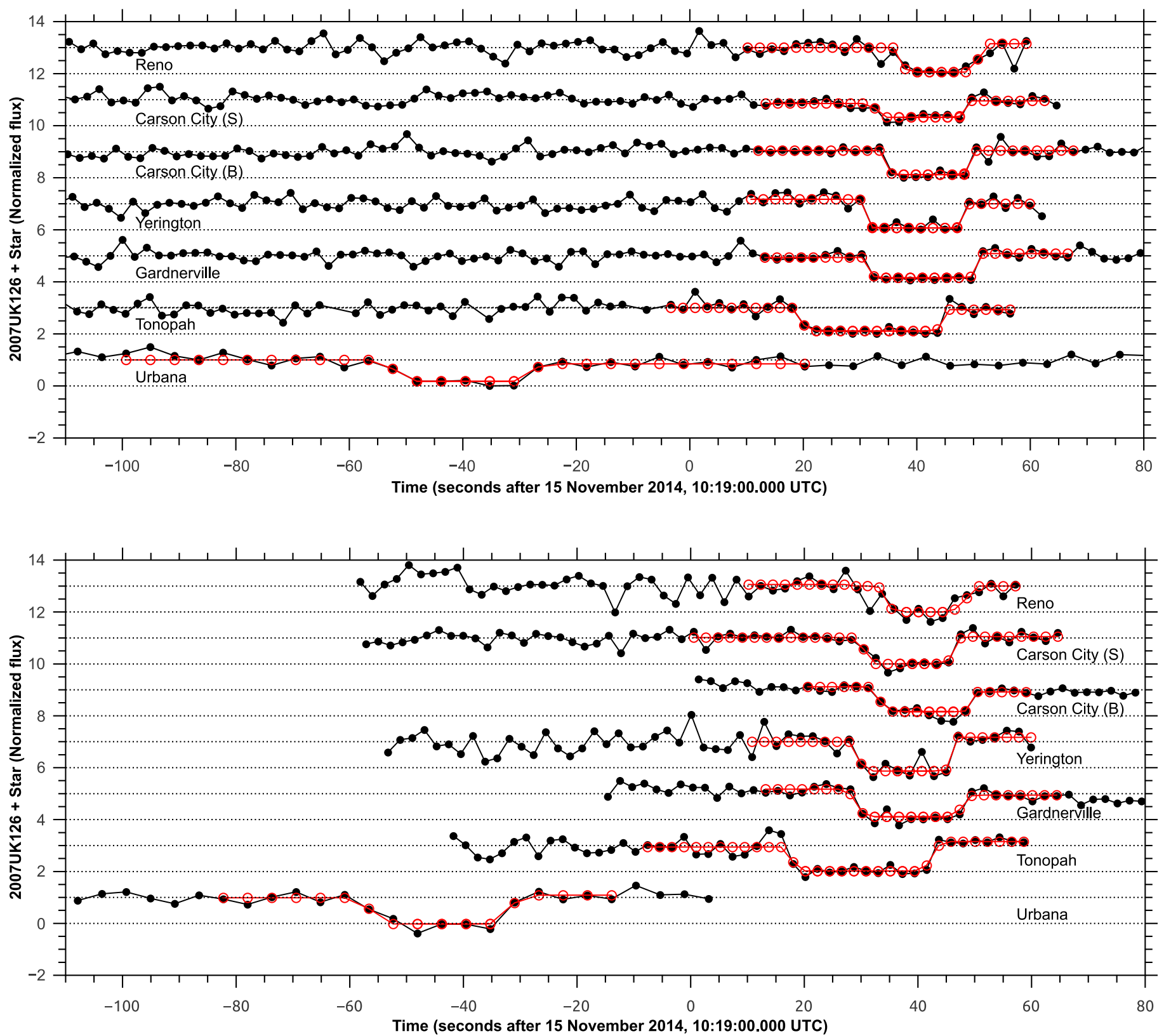

Figure 2. Top panel: the seven occultation light curves, obtained from the GBR extraction (see text), normalized to the unocculted star plus $2007 \mathrm{UK}_{126}$ flux and vertically shifted by integer values for better viewing. The red lines and circles are the square-well model convoluted by the Fresnel diffraction, the star apparent angular diameter, and the finite exposure time. The mid-times of the occultations do not coincide due to the propagation delays of the shadow due to the distinct longitudes of the sites (Figure 1). Bottom panel: same from the MWB extraction. Note that no secondary occultation is observed, as could be caused by a satellite.

with $\Delta m=0.03 \pm 0.01 \mathrm{mag}$ (Thirouin et al. 2014)). Thus, we will assume here that this TNO is close to the Maclaurin state.

Consequently, its limb is elliptical and is characterized by $M=5$ adjustable parameters: the coordinates of the body center, relative to the star in the plane of the sky $\left(f_{c}, g_{c}\right)$; the apparent semimajor axis $a^{\prime}$; the apparent oblateness $\epsilon^{\prime}=\left(a^{\prime}-b^{\prime}\right) / a^{\prime}$ (where $b^{\prime}$ is the apparent semi-minor axis); and the position angle (PA) of the semi-minor axis $b^{\prime}$. The PA is counted positively from the direction of celestial north to celestial east, while the quantities $\left(f_{c}, g_{c}\right)$ are expressed in kilometers, positively toward the celestial east and north directions, respectively. In the oblate Maclaurin spheroid hypothesis, the apparent oblateness $\epsilon^{\prime}$ is related to the true oblateness $\epsilon=1-(c / a)$ (where $a=a^{\prime}$ and $c$ are the true equatorial and polar radii, respectively) through

$$
\epsilon^{\prime}=1-\sqrt{\cos ^{2}(\xi)+(1-\epsilon)^{2} \sin ^{2}(\xi)},
$$

where $\xi$ is the polar aspect angle, i.e., the angle between the polar $c$-axis and the line of sight. The case $\xi=0^{\circ}$ (respectively $\xi=90^{\circ}$ ) then corresponds to the pole-on (respectively equatoron) geometry.

Note that $2007 \mathrm{UK}_{126}$ 's pole direction is currently unknown, so $\xi$ is undefined. Finally, it is useful to quantify the size of the body through its apparent equivalent radius $R_{\mathrm{eq}}$ (rather than its equatorial radius $a$ ) defined by $R_{\mathrm{eq}}=\sqrt{a^{\prime} b^{\prime}}=a^{\prime} \sqrt{1-\epsilon^{\prime}}$. This corresponds to the radius of the disk that has the same area as that enclosed by the apparent limb. 


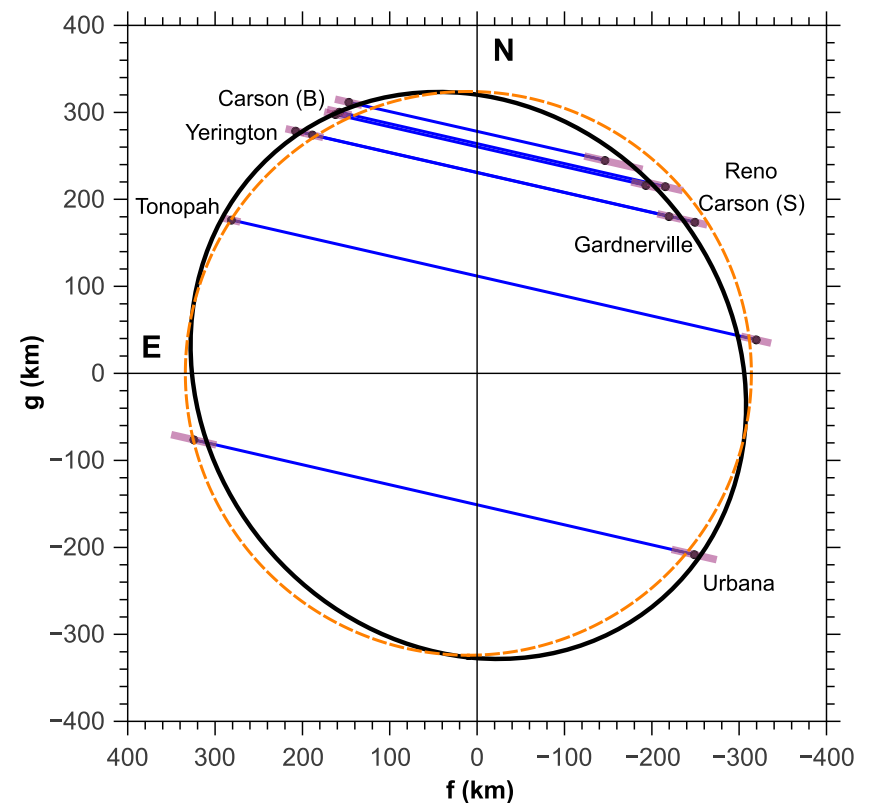

Figure 3. Positive occultation chords and the GBR solution (see text). The best elliptical fit to the occultation chords are shown in black and the dashed orange line is the circular fit. Adjusted parameters are shown in Table 4. The magenta segments are the 1- $\sigma$ error bars on each occultation chord extremity.

The seven positive occultation chords provide $N=14$ data points (the ingress and egress chord extremities, see Figure 3 and Table 3 ), whose positions are denoted as $f_{i, \text { obs }}, g_{i, \text { obs }}$. The best elliptical fit to those points minimizes the radial residuals, from which the relevant $\chi^{2}$ function is defined. The quality of the fit is then assessed through the value of the $\chi^{2}$ function per degree of freedom (or unbiased $\chi^{2}$ ), defined as $\chi_{\text {pdf }}^{2}=\chi^{2} /(N-M)$; see, e.g., Sicardy et al. (2011) for details. The 1- $\sigma$ level uncertainty of a given parameter is then obtained by varying it (all the other parameters being readjusted during this exploration) so that the $\chi^{2}$ function varies from its minimum value $\chi_{\min }^{2}$ to $\chi_{\min }^{2}+1$.

\section{RESULTS}

Figures 3 and 4 display the best elliptical limb fits obtained from the two sets of timings (GBR and MWB). Note that the difference between the immersion and emersion times comes from the fact that the determination of the instants is very sensitive to the photometry. Since the star is very faint and its flux is close to the background sky flux, a small change on the light curve can induce different instants.

Although having slightly different times, the parameters derived from each method agree with each other at the 1- $\sigma$ level (Table 4). Moreover, the respective values of $\chi_{\mathrm{pdf}}^{2}$ for the elliptical fits are under 0.7 , indicating satisfactory adjustements of the model to the data.

Purely circular shapes were also fitted to the data, resulting in $\chi_{\mathrm{pdf}}^{2}$ values of 1.69 and 1.46 for the GBR and MWB timings, respectively. Thus, although circular fits remain acceptable in terms of quality, they do degrade the value of $\chi_{\mathrm{pdf}}^{2}$. In fact, Table 4 shows that the apparent oblatenesses $\epsilon^{\prime}$ differ from zero at the $\sim 2.5-\sigma$ level, a marginal detection of non-sphericity for $2007 \mathrm{UK}_{126}$.

Note that in both solutions the Urbana chord seems a bit displaced toward east (Figures 3 and 4), compared to the

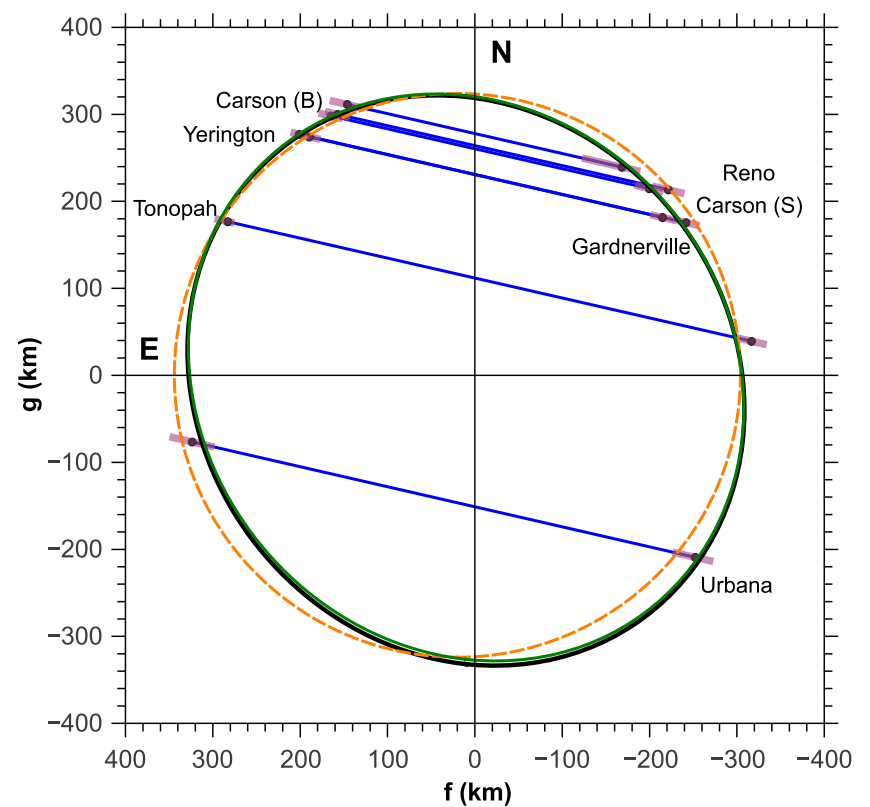

Figure 4. Same as Figure 3 for the MWB solution. The best elliptical fit is in black and the fit from GBR is shown in green. Note that the two solutions are very close to each other, with a maximum radial discrepancy of about $5 \mathrm{~km}$.

Table 4

Physical Parameters of 2007 UK $_{126}$ from the Two Studied Solutions

\begin{tabular}{lcc}
\hline \hline Solution & GBR & MWB \\
\hline Semimajor axis (km) & $\mathbf{3 3 9}_{-10}^{+15}$ & $340_{-8}^{+12}$ \\
Equivalent radius (km) & $319_{-7}^{+14}$ & $319_{-6}^{+12}$ \\
Circular fit radius (km) & $324_{-23}^{+27}$ & $328_{-21}^{+26}$ \\
Apparent oblateness & $\mathbf{0 . 1 0 6}_{-0.040}^{+0.050}$ & $0.118_{-0.048}^{+0.055}$ \\
$f_{c}(\mathrm{~km})$ & $-3699 \pm 12$ & $-3699 \pm 13$ \\
$g_{c}(\mathrm{~km})$ & $-3457 \pm 13$ & $-3456 \pm 13$ \\
Position angle (deg) & $129_{-22}^{+14}$ & $134_{-17}^{+14}$ \\
$\chi_{\text {pdf }}^{2}($ elliptical fit) & $\mathbf{0 . 7 1}$ & $\mathbf{0 . 6 5}$ \\
$\chi_{\text {pdf }}^{2}($ circular fit) & 1.65 & 1.45 \\
Radial rms (km) & 10.6 & 10.9 \\
$p_{R}($ Thirouin) & $0.189_{-0.015}^{+0.009}$ & $0.189_{-0.013}^{+0.008}$ \\
$p_{V}($ Perna) & $0.159_{-0.013}^{+0.007}$ & $0.159_{-0.011}^{+0.006}$ \\
Density (kg m $\left.{ }^{-3}\right)^{\mathrm{c}}$ & $<1740$ & $<1620$ \\
\hline
\end{tabular}

Notes.

a " $p_{R}$ (Thirouin)" means $p_{R}$ using $H$ from MPC and Thirouin et al. (2014).

b " $p_{V}$ (Perna)" means $p_{V}$ using $H$ from Perna et al. (2010).

${ }^{c}$ Upper limit based on a hydrostatic shape with a rotation period of $11.05 \mathrm{hr}$.

elliptic model. Since the TNO is near the limit of the critical range to be a Maclaurin object, we can consider two possibilities: (1) all the timings are correct within $1 \sigma$ uncertainty, implying that $2007 \mathrm{UK}_{126}$ is compatible with a Maclaurin object and has large topographic features (craters or mountains) of the order of $10 \mathrm{~s}$ of kilometers (a possible solution, considering the New Horizons images on Charon that shows topographic features as big as $+/-6 \mathrm{~km} \mathrm{Nimmo} \mathrm{et} \mathrm{al.}$ 2016) or (2) $2007 \mathrm{UK}_{126}$ is a smooth (no topographic features) Maclaurin object, implying that timing problems are present at some stations.

With this in mind, we have allowed time shifts for all stations, aligning the middles of all chords, resulting in a $\chi_{\mathrm{pdf}}^{2}$ value of 1.37 and a radial $\mathrm{rms}$ of $16 \mathrm{~km}$, i.e., without significant 
improvement of the fit quality. Moreover, this implies that all sites had timing issues, some of them as big as one second (half of the integration time), which is unlikely to happen.

The fact that the observations are not repeatable makes difficult the assessment of timing errors. However, we do not expect large absolute timing errors at the various stations. Although it is important to note that the process of converting video to the stack images on GBR analysis (Section 3.1.1) may present some small error in time. For the MWB analysis, the conversion from video data to a light curve does not present any intrinsic timing errors beyond limitations imposed by the low signal-to-noise ration for the event (Section 3.1.2).

\subsection{Physical Properties for $2007 U_{126}$}

From the size and shape, the density can be derived if the mass of the system is known, e.g., through the motion of a satelite. As mentioned in Section 1, Grundy et al. (2011) reported the discovery of a companion, but no orbital elements are presently available for it. However, constraints on the density can still be derived in the Maclaurin hypothesis, when combined to the rotation period $P$, using the equilibrium equation (Plummer 1919):

$$
\rho=\frac{4 \pi \sin ^{3}(\theta)}{G \cos (\theta) \cdot\{2 \theta[2+\cos (2 \theta)]-3 \cdot \sin (2 \theta)\} \cdot P^{2}},
$$

where $G$ is the gravitational constant $(G=$ $6.67408 \cdot 10^{-11} \mathrm{~m}^{3} \mathrm{~kg}^{-1} \mathrm{~s}^{-2}$ ) and $\theta$ is related to the real oblatenes, $\epsilon$, by $\cos (\theta)=1-\epsilon$.

Equation (2) imposes that the true oblateness $\epsilon$ is bounded according to $\epsilon^{\prime} \leqslant \epsilon$. On the other hand, Maclaurin spheroids can have a maximum oblateness of $\epsilon=0.417$. For $\epsilon>0.417$, only triaxial ellipsoid of equilibrium are possible (Chandrasekhar 1987). Moreover, the polar aspect angle must satisfy $\arcsin \left(\sqrt{\left(\left(1-\epsilon^{\prime}\right)^{2}-1\right) /\left((1-\epsilon)^{2}-1\right)}\right) \leqslant \xi \leqslant \pi / 2$, the lower limit case corresponding to $\epsilon=0.417$ and the upper limit case corresponding to $\epsilon=\epsilon^{\prime}$. In that context, it is interesting to assess the density of probability for $\epsilon$, beyond merely stating that it should lie in the interval $\left[\epsilon^{\prime}, 0.417\right]$.

For an elliptical fit the orientation of 2007 UK $_{126}$ 's pole angle is partially constrained by the ellipse orientation. The polar aspect angle $\xi$ remains undetermined but constrained to an interval $[0.45, \pi / 2]$ through Equation (2) and constraints on $\epsilon$. Because the PA of the fitted elliptical limb is known (Table 4), we can assume here that the density of probability for $\xi$ is uniformly distributed over all the possible values given by the interval.

Consequently, the probability to have $\xi$ in the interval is $\sim 0.7$, considering the possible values of $\epsilon^{\prime}$ (Table 4). In other words, the measured apparent oblateness $\epsilon^{\prime}$ does not require a very specific, fine-tuned orientation for the aspect angle.

Using both the GBR and MWB solutions we obtain a lowest possible value for $\epsilon^{\prime}$ (i.e., $0.105-0.0040=0.065$ ) from which we can derive an upper limit for the density of $\rho=1740 \mathrm{~kg} \mathrm{~m}^{-3}$.

The preferred rotation period for $2007 \mathrm{UK}_{126}$ is $P=11.05 \mathrm{hr}$ (Thirouin et al. 2014). However, other possible aliases exist, and only a secure lower limit of $P>8 \mathrm{hr}$ is eventually given by those authors. Figure 5 presents the Maclaurin equilibrium curve for both periods.

To numerically estimate the probability distribution, $P_{r}(\rho)$, for the density, we generate a sample of aspect angles from an uniform distribution in the valid interval. This in turn provides

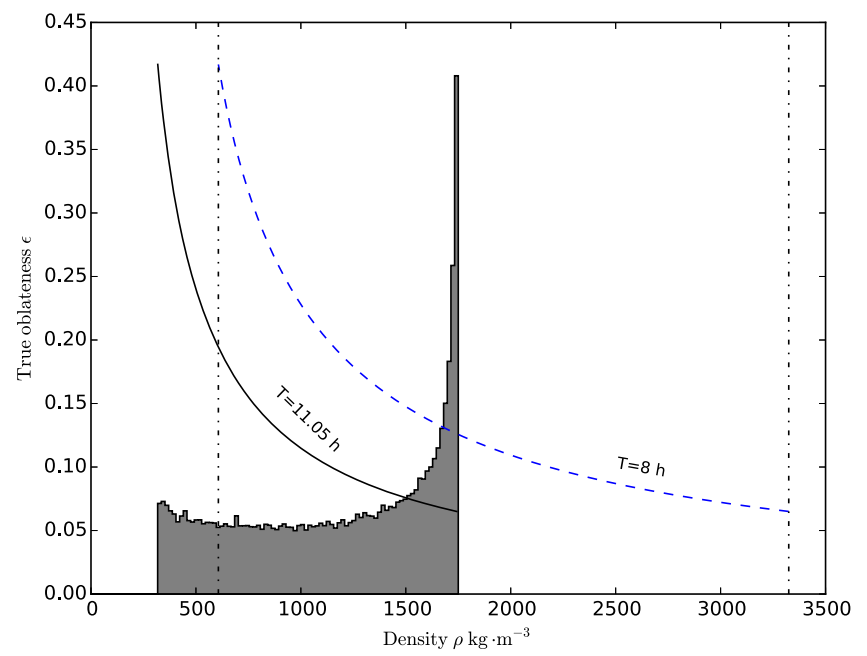

Figure 5. Continuous line is the Maclaurin equilibrium curve for the preferred period of $11.05 \mathrm{hr}$. The lower limit is given by the stability condition for a Maclaurin spheroid, while the upper limit is given by the occultation. The grayfilled histogram is the probability distribution for the density within this range in arbitrary units. The dashed blue line is the oblateness density relation for a period $T=8 \mathrm{hr}$, the lowest probable rotation period (see text). In this case, the probability distribution is qualitatively the same (not plotted) but in the range between 600 and $3300 \mathrm{~kg} \mathrm{~m}^{-3}$ indicated by vertical dash-dotted lines.

the density of probability for the density via Equations (2) and (3). We use the lowest $1-\sigma$ value for $\epsilon^{\prime}=0.065$ and the prefered value for $T=11.05 \mathrm{hr}$.

Figure 5 also displays $P_{r}(\rho)$. It shows that although $\rho$ can be in the whole range $[320,1740] \mathrm{kg} \mathrm{m}^{-3}$ it is probably close to its upper limit, indicating an icy body. For a lowest rotation period of $P=8 \mathrm{hr}$, the probability distribution for the density will be qualitatively the same but in the range $[600,3300] \mathrm{kg} \mathrm{m}^{-3}$ indicated in Figure 5 for reference. In this case the density is probably close to its upper limit of $3300 \mathrm{~kg} \mathrm{~m}^{-3}$, indicating a rocky body. Only with an accurate rotation period measurement can a more definitive conclusion for the density be stated.

From the equivalent radius $R_{\text {eq }}$, we obtain the geometric albedo $p$ :

$$
p=\left(A U_{k m} / R_{\mathrm{eq}}\right)^{2} \times 10^{0.4\left(H_{\odot}-H\right)},
$$

where $A U_{\mathrm{km}}=1.49598 \times 10^{8} \mathrm{~km}, H_{\odot}$ is the Sun magnitude at $1 \mathrm{AU}\left(H_{\odot, V}=-26.74\right)$, and $H$ is the object absolute magnitude of the object. The Minor Planet Center provides $H_{R}=3.4$ and Perna et al. $(2010,2013)$ give $H_{V}=3.59 \pm 0.04$. Adopting the ranges of equivalent radii obtained for both solutions, we calculate the geometric albedo of $2007 \mathrm{UK}_{126}$ in the visible $\left(p_{V}\right.$ and $p_{R}$ ). The error bars represent the range of the albedo obtained for a given solution, combined with the uncertainty in absolute magnitude. Results are presented in Table 4.

\section{CONCLUSION}

We observed the first multi-chord stellar occultations by the trans-Neptunian object (229762) $2007 \mathrm{UK}_{126}$. The shadow crossed the United States on 2014 November 15 and we obtained 7 positive chords, from which we obtain the equivalent radius and geometric albedo of the body, and an upper limit for its density.

This is the first TNO occultation result from the RECON project. It was observed during the pilot phase with only a few sites in operation. For future occultations, with sufficient 
astrometric support, five times as many RECON sites will be available for observations.

We present in this paper two independent analyses that provide consistent solutions for 2007 UK126's limb shape. The GBR solution gives an equivalent radius $R_{\mathrm{eq}}=319_{-7}^{+14} \mathrm{~km}$ and geometric albedo that may vary $p_{V}=0.159_{-0.013}^{+0.007}$ to $p_{R}=0.189_{-0.015}^{+0.009}$, depending on the adopted absolute magnitude. The MWB solution provides an equivalent radius of $R_{\text {eq }}=319_{-6}^{+12} \mathrm{~km}$ and albedo that may vary from $p_{V}=$ $0.159_{-0.011}^{+0.006}$ to $p_{R}=0.189_{-0.013}^{+0.008}$. The two solutions give comparable minimum $\chi^{2}$ per degree of freedom $(0.59$ and 0.56 , respectively). The equivalent radii we derive here are consistent with, but more accurate than, the value based on Herschel observations, $R_{e q}=299.5 \pm 38.5 \mathrm{~km}$ (Santos-Sanz et al. 2012).

A range for the density $\rho$ was estimated to be [318, $1740] \mathrm{kg} \mathrm{m}^{-3}$ using a lowest apparent oblateness from the $G B R$ solution and considering the rotation period of $11.05 \mathrm{hr}$. Those values are comparable to other TNOs densities found in literature (as presented in Brown 2012; Santos-Sanz et al. 2016 and references). No other information could be derived since there is insufficient orbital information available. Obtaining an orbital solution for the satellite of $2007 \mathrm{UK}_{126}$ would be an importnat step foward, as it would firmly constrain the mass of the primary, and thus, its density from our size measurement.

The occultation chords also shows that there is no evidence of a very close binary and no satellite could be detected from the shadow track direction.

The authors want to thank Barclay Anderson, Charley Arrowsmith, Buck Bateson, Clair Blackburn, Teralyn Blackburn, Mystery Brown, Brian Cain, Mark Callahan, Shelley Callahan, Matt Christiansen, Lynn Coffman, Brian Crosby, Scott Darrington, Adam Eisenbarth, Bill Gimple, Erick Hsieh, Todd Hunt, Levi Kinateder, Colton Kohler, Joanna Kuzia, Les Kuzia, Ethan Lopes, Ian Mahaffey, Jason Matkins, Andrew Maynesik, Terry Miller, Seth Nuti, Melanie Phillips, Jiawei Simon Qin, Jim Reichle, Dan Ruby, Jeff Schloetter, David Schulz, Jeannie Smith, Kathy Trujillo, Jacob Wagner, Andrew Yoder, Ted Zel, and all people involved in this occultation observation.

The RECON project would not be possible without all of the support from our community teams (teachers, students, and community members) and was funded by NSF grants AST1212159, AST-1413287, and AST-1413072. Special thanks to Dean and Starizona for their support of RECON above and beyond the usual bounds of a commercial relationship.

Part of the research leading to these results has received funding from the European Research Council under the European Community's H2020 (2014-2020/ERC Grant Agreement n 669416 "LUCKY STAR").

Funding from Spanish grant AYA-2014-56637-C2-1-P is acknowledged, as is the Proyecto de Excelencia de la Junta de Andaluca, J. A. 2012-FQM1776. R.D. acknowledges the support of MINECO for his Ramon y Cajal Contract. FEDER funds are also acknowledged.

The authors want to thank the partial use of OPD/LNA facilities for this work.

A.D.O. is thankful for the support of the CAPES (BEX 9110/12-7) FAPERJ/PAPDRJ (E-26/200.464/2015) grants.

A.R.G.J. thanks CAPES/Brazil.
G.B.R. is thankful for the support of CAPES/Brazil and FAPERJ (Grant E-01/2015/209545).

J.I.B.C. acknowledges CNPq for a PQ2 fellowship (process number 308489/2013-6).

L.G. thanks the support from CONACYT through grant 167236.

M.A. thanks the CNPq (Grants 473002/2013-2 and 308721/ 2011-0) and FAPERJ (Grant E-26/111.488/2013).

P.S.-S. acknowledges that part of the research leading to these results has received funding from the European Unions Horizon 2020 Research and Innovation Programme, under Grant Agreement no 687378.

R.V.M. acknowledges the following grants: CNPq-306885/ 2013, CAPES/Cofecub-2506/2015, FAPERJ/PAPDRJ-45/ 2013, FAPERJ/CNE/05-2015.

This research was partially based on data obtained at the Lowell Observatory's Discovery Channel Telescope (DCT). Lowell operates the DCT in partnership with Boston University, Northern Arizona University, the University of Maryland, and the University of Toledo. Partial support of the DCT was provided by Discovery Communications. LMI was built by Lowell Observatory using funds from the National Science Foundation (AST-1005313). A.T., S.L., and B.S. acknowledge Lowell Observatory funding.

The MEarth project acknowledges funding from the National Science Foundation, the David and Lucile Packard Foundation Fellowship for Science and Engineering, and the John Templeton Foundation. The opinions expressed here are those of the authors and do not necessarily reflect the views of the John Templeton Foundation.

\section{REFERENCES}

Alvarez-Candal, A., Ortiz, J. L., Morales, N., et al. 2014, A\&A, 571, 48 Assafin, M., Camargo, J. I. B., Vieira Martins, R., et al. 2010, A\&A, 515, 32 Assafin, M., Camargo, J. I. B., Vieira Martins, R., et al. 2012, A\&A, 541, 142 Assafin, M., Vieira Martins, R., Camargo, J. I. B., et al. 2011, Gaia Follow-up Network for the Solar System Objects: Gaia FUN-SSO Workshop Proc., ed. P. Tanga \& W. Thuillot 85

Audela Software 2016, Online: http://audela.org/

Braga Ribas, F., Sicardy, B., Ortiz, J. L., et al. 2012, in AAS Meeting 44 Abstracts \#402.01

Braga-Ribas, F., Sicardy, B., Colas, F., et al. 2011, CBET, 2675, 1

Braga-Ribas, F., Sicardy, B., Ortiz, J. L., et al. 2013, ApJ, 773, 26

Braga-Ribas, F., Sicardy, B., Ortiz, J. L., et al. 2014, Natur, 508, 72

Brown, M. E. 2012, AREPS, 40, 467

Buie, M. W., \& Keller, J. M. 2016, AJ, 151, 73

Camargo, J. I. B., Vieira-Martins, R., Assafin, M., et al. 2014, A\&A, 561, 37 Chandrasekhar, S. 1987, Ellipsoidal figures of equilibrium (New York: Dover) Desmars, J., Camargo, J. I. B., Braga-Ribas, F., et al. 2015, A\&A, 584, A96 Elliot, J. L., Person, M. J., Zuluaga, C. A., et al. 2010, Natur, 465, 897

Gladman, B., Marsden, B. G., \& Vanlaerhoven, C. 2008, in The Solar System Beyond Neptune, Nomenclature in the Outer Solar System, ed. M. A. Barucci et al. (Tucson, AZ: Univ. Arizona Press), 43

Grundy, W. M., Benecchi, S. D., Buie, M. W., et al. 2011, in EPSC-DPS Joint Meeting 2011, 1078

JPL Small-Body Database Browser 2016, Online: http://ssd.jpl.nasa.gov/ sbdb.cgi

Lykawka, P. S., \& Mukai, T. 2008, AJ, 135, 1161

Minor Planet Center-List Of Transneptunian Objects 2016, Online: http:// www.minorplanetcenter.net/iau/lists/TNOs.html

Minor Planet Center-List Of Centaurs and Scattered-Disk Objects 2016, Online: http://www.minorplanetcenter.net/iau/lists/Centaurs.html Nimmo, F., Umurhan, O. M., Lisse, C. M., et al. 2016, arXiv:1603.00821 Ortiz, J. L., Duffard, R., Pinilla-Alonso, N., et al. 2015, A\&A, 576, 180 Ortiz, J. L., Sicardy, B., Braga-Ribas, F., et al. 2012, Natur, 491, 5660 Parker, A., Pinilla-Alonso, N., Santos-Sanz, P., et al. 2015, arXiv:1511.01112 Perna, D., Barucci, M. A., Fornasier, S., et al. 2010, A\&A, 510, 53 Perna, D., Dotto, E., Barucci, M. A., et al. 2013, A\&A, 554, 49 
Person, M. J., Elliot, J. L., Bosh, A. S., et al. 2011, in AAS Meeting 218 Abstracts \#224.12

Plummer, H. C. 1919, MNRAS, 80, 26

Ruprecht, J. D., Bosh, A. S., Person, M. J., et al. 2015, Icar, 252, 271

Sallum, S., Brothers, T., Elliot, J. L., et al. 2011, in AAS Meeting 218 Abstracts \#224.13

Santos-Sanz, P., French, R. G., \& Pinilla-Alonso, N. 2016, PASP, 128, 959

Santos-Sanz, P., Lellouch, E., Fornasier, S., et al. 2012, A\&A, 541, A92

Schwamb, M. E., Brown, M. E., Rabinowitz, D., \& Marsden, B. G. 2008, MPEC, 2008, D38

Sicardy, B., Colas, F., Maquet, L., et al. 2010, in AAS Meeting 42 Abstracts \#23.11
Sicardy, B., Ortiz, J. L., Assafin, M., et al. 2011, Natur, 478, 493

Stansberry, J., Grundy, W., Brown, M., et al. 2008, in The Solar System Beyond Neptune, Physical Properties of Kuiper Belt and Centaur Objects: Constraints from the Spitzer Space Telescope, ed. M. A. Barucci et al. (Tucson, AZ: Univ. Arizona Press), 161

Tancredi, G., \& Favre, S. 2008, Icar, 195, 851

Thirouin, A., Noll, K. S., Ortiz, J. L., \& Morales, N. 2014, A\&A, 569, 3 van Belle, G. T. 1999, PASP, 111, 1515

Widemann, T., Sicardy, B., Dusser, R., et al. 2009, Icar, 199, 458

Zacharias, N., Monet, D. G., Levine, S. E., et al. 2004, in AAS Meeting 205 Abstracts \#48.15 